Artículo

\title{
Tasa de aireación de la degradación aerobia en la fracción orgánica de residuos sólidos urbanos
}

\author{
Gabriela Carrillo-Sancen ${ }^{1}$ \\ Manuel Alejandro Cuautle-Marin ${ }^{2}$ \\ Francisco Javier Martínez-Valdez ${ }^{1 \S}$ \\ Gerardo Saucedo-Castañeda ${ }^{3}$ \\ Dimitrios Komilis ${ }^{4}$
}

${ }^{1}$ División Químico Biológicas-Universidad Tecnológica de Tecámac. Tecámac, Estado de México, México. CP. 55740. (gcarrilloS@uttecamac.edu.mx). ${ }^{2}$ Laboratorio de investigación en procesos avanzados de tratamiento de aguas-Instituto de Ingeniería-Unidad Académica Juriquilla-Universidad Nacional Autónoma de México. Blvd. Juriquilla 3001, Querétaro, México. (macmarin@live.com.mx). ${ }^{3}$ Departamento de Biotecnología-Universidad Autónoma Metropolitana-Unidad Iztapalapa. México. CP. 09340. (saucedo@xanum.uam.mx). ${ }^{4}$ Departamento de Ingeniería Ambiental-Universidad Demócrito de Tracia. Vasilissis Sofias 12, Xanthi 671 00, Grecia. (dkomilis@env.duth.gr).

${ }^{\S}$ Autor para correspondencia: fmartinezv@uttecamac.edu.mx.

\section{Resumen}

La cinética microbiana y enzimática son factores importantes durante la degradación aerobia de la fracción orgánica de los residuos sólidos urbanos, estas dependen principalmente de la temperatura de incubación y las tasas de aireación. El objetivo de esta investigación fue evaluar el proceso de degradación aerobia, por múltiples variables y su combinación para comprender las interacciones entre las tasas de aireación en la degradación aerobia y sus respuestas. Las tasas de aireación se fijaron en $0.032,0.064,0.125,0.251$ y $0.392 \mathrm{~L}$ de aire húmedo $\mathrm{kg}^{-1} \mathrm{~min}^{-1}$ a $35^{\circ} \mathrm{C}$ con inóculo. La actividad microbiana se evaluó de forma indirecta por medio de la respirometria; es decir, la generación de $\mathrm{CO}_{2}$ y el consumo de $\mathrm{O}_{2}$. Las actividades enzimáticas extracelulares (es decir, pectinasas, celulasas, xilanasas y proteasas) se cuantificaron mediante la liberación de los azúcares reductores. Los diferentes ensayos se realizaron en la Universidad Autónoma Metropolitana Unidad Iztapalapa en septiembre de 2019. Encontrando una fuerte relación positiva entre la actividad enzimática xilanasa y pectinasa con la pérdida de peso en seco, junto con el aumento de las actividades celulasas y xilanasas a mayores tasas de aireación.

Palabras clave: actividades enzimáticas, respirometría, tasa de aireación.

Recibido: junio de 2021

Aceptado: agosto de 2021 


\section{Introducción}

El oxígeno es esencial para la actividad microbiana durante la degradación aerobia (Guo et al., 2012). Una concentración mínima de oxígeno de 5\% (v/v) en el centro de la pila de compostaje es necesaria para asegurar las condiciones aerobias (Rasapoor et al., 2009). La tasa de aireación óptima depende de la composición de las materias primas y de los métodos de ventilación (Komilis et al., 2011). Si la aireación se expresa como intensidad de flujo; es decir, flujo de aire por unidad de masa de sustrato, se obtiene una variable independiente de la escala (Rodríguez-Fernández et al., 2012). Esto, en la fermentación en medio sólido, se expresa como el promedio de la tasa de flujo de aire (L) por kilogramo de sustrato por tiempo ( $\mathrm{min})\left(\mathrm{v} \mathrm{kg}^{-1} \mathrm{~min}^{-1} \mathrm{o} \mathrm{vkgm}\right.$, como se utilizará en el escrito).

Los gradientes de temperatura y humedad de la masa sólida deben ser controlados para lograr un proceso satisfactorio (Martins et al., 2011). El intervalo de temperaturas entre $35-40{ }^{\circ} \mathrm{C}$ favorece el desarrollo de un consorcio microbiano diverso (Saludes et al., 2007). El aumento de la temperatura en la pila de compostaje está relacionado con el aumento de la actividad microbiana (Raut et al., 2008; Li et al., 2013). La calidad (estabilidad y madurez) de la composta está determinada por múltiples variables o su combinación (Bernal et al., 2009).

Varios autores han intentado desarrollar modelos matemáticos, estocásticos y estadísticos para comprender las múltiples relaciones e interacciones entre las condiciones operacionales y las respuestas del compostaje (Li et al., 2013). Se han utilizado métodos estadísticos multivariantes para procesar datos del proceso de digestión anaerobia, en los que se intenta correlacionar la composición bioquímica con los rendimientos de metano (Bayard et al., 2015; Liu et al., 2015; El Achkar et al., 2017). El uso combinado de análisis de componentes principales (ACP), análisis de conglomerados y pruebas de biodegradabilidad puede ayudar a correlacionar los niveles de nutrientes y la actividad microbiana en la digestión anaerobia (Gil et al., 2018).

Durante los procesos de compostaje, el análisis de ACP se ha aplicado ampliamente para clasificar la generación de malos olores y su correlación con los diferentes residuos orgánicos y su composición (Toledo et al., 2018). Los algoritmos de ACP emplean parámetros fisicoquímicos tanto en los procesos aerobios como en los anaerobios. El proceso de compostaje puede caracterizarse bien por la actividad enzimática extracelular (Tiquia et al., 2002). Con base en lo anterior, el objetivo de este trabajo fue evaluar el proceso de degradación aerobia en función de diversas variables para modelar las relaciones e interacciones entre las tasas de aiereación durante el compostaje y las variables de respuesta como lo son: el consumo de oxígeno $\left(\mathrm{O}_{2}\right)$, la generación de dióxido de carbono $\left(\mathrm{CO}_{2}\right)$, el consumo de azúcares reductores y las actividades enzimáticas xilanasas, celulasas, proteasas y pectinasas.

\section{Materiales y métodos}

\section{Sustratos e inóculo}

La fracción orgánica de los residuos sólidos urbanos (FORSU) se obtuvo de los establecimientos de elaboración de alimentos en Iztapalapa, en la Ciudad de México (México). La composta estable de la planta de compostaje de residuos sólidos urbanos mezclados del Bordo Poniente de la Ciudad de México (PCBP) se utilizó como fuente de consorcios microbianos (CM) como inoculo. La toma de composta de la PCBP se llevó acabo de acuerdo con la norma ASTM D-34 (2016). 


\section{Estrategia experimental}

La degradación de la FORSU se realizó en cuatro biorreactores tubulares (BT) con capacidades de $0.98,1.91$ y $3.84 \mathrm{~L}$. Los BT se empacaron con una mezcla en base húmeda de: FORSU 83\%, consorcio microbiano $8 \%$, aserrín $4 \%$, papel $3 \%$ y poda $2 \%$ para lograr una relación C/N inicial de 30 (Martínez-Valdez et al., 2015). La humedad inicial fue de 70\% (Liang et al., 2003). Durante la degradación aerobia, las tasas de aireación se incrementaron en una proporción de 0.5 en los diferentes experimentos $(0.032,0.064,0.125,0.251$ y $0.392 \mathrm{vkgm})$. El sistema de respirometría fue operado de acuerdo con Saucedo-Castañeda et al. (2013); Torres-Mancera et al. (2018). La calibración del equipo de respirometría se hizo con mezclas estándar de $\mathrm{CO}_{2}$ y $\mathrm{O}_{2}$.

\section{Identificación de microorganismos}

Todos los BT se inocularon (composta de PCBP) y los ensayos se realizaron a $35^{\circ} \mathrm{C}$ debido a la mayor actividad microbiana (mostrada por respirometría) a esa temperatura (Martínez-Valdez et al., 2015). Una vez completado el proceso, se desempacaron los BT, se pesó el material en una balanza analítica (Ohaus Galaxy ${ }^{\circledR}$ 200) y se determinó la humedad, pH y actividad enzimática. Todas las muestras se obtuvieron en el momento de máxima tasa de generación de $\mathrm{CO}_{2}$, ya que esto indicaba la mayor actividad microbiana.

El análisis de la población microbiana se realizó mediante la técnica de electroforesis en gel de gradiente desnaturalizante (EGGD): se tomaron muestras en todas las condiciones y se centrifugaron a $8.049 .6 \mathrm{~g}$. Se tomo el precipitado $(1 \mathrm{~g})$ y se procedió a la extracción y purificación del ADN utilizando el kit de aislamiento de ADN PowerSoil ${ }^{\circledR}$ (MoBio). Una vez realizada la extracción y la purificación, se corrió un gel de agarosa al 1\% para corroborar el éxito de la extracción de ADN. Posteriormente, se realizó una reacción en cadena de la polimerasa $(\mathrm{RCP})$ para obtener copias in vitro de un fragmento de la secuencia del gen del ARNr 16S de cada una de las muestras, que se llevó a cabo en un termociclador (Multigene, Labnet International, Inc.).

La mezcla de la reacción, con un volumen final de $50 \mu \mathrm{L}$, contiene $10 \mathrm{X}$ de amortiguador, 1.5 mmol de $\mathrm{MgCl}_{2}, 200 \mu \mathrm{m}$ de cada dNTP (Promega Corporation, EE. UU), $10 \mathrm{pM}$ de cada iniciador,

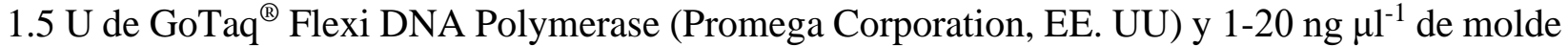
de ADN. Las condiciones de amplificación fueron las siguientes: 2 min a $95{ }^{\circ} \mathrm{C}$ de desnaturalización inicial, 25 ciclos que constaban de $30 \mathrm{~s}$ a $95^{\circ} \mathrm{C}, 30$ segundos a $50{ }^{\circ} \mathrm{C}$ y $1 \mathrm{~min}$ a $72{ }^{\circ} \mathrm{C}$, seguidos de una extensión final de 5 min a $72^{\circ} \mathrm{C}$. Los amplicones obtenidos se almacenaron a $4{ }^{\circ} \mathrm{C}$ después de su corrida por electroforesis $(100 \mathrm{~V}, 30 \mathrm{~min})$ en un gel de agarosa al $1 \%$. Después de la amplificación de la secuencia del gen 16S rRNA, se hizo una nueva amplificación anidada usando el amplicón obtenido previamente como molde de ADN.

Los iniciadores 1070F (5' ATG GCT GTC GTC AGC T 3') y 1392R+GC (5' CGC CCG CCG CGC CGC CCC GCC GCC GCC GCC GCC CCG CCC CCC ACG GGC GGR GRG TAC 3’) se utilizaron con las mismas condiciones de amplificación cambiando la temperatura de alineación a $55^{\circ} \mathrm{C}$. Las muestras se purificaron con un kit comercial (Wizard ${ }^{\circledR}$ SV Gel y Sistema de limpieza de PCR, Promega Corporation) siguiendo el protocolo descrito por el proveedor. La cuantificación de ácidos nucleicos de ADN se midió con el espectrofotómetro NanoDrop ND1000 (Thermo Scientific). Había $1 \mu \mathrm{l}$ con $500 \mathrm{ng}$ de ADN de cada muestra añadida a $5 \mu \mathrm{l}$ de amortiguador de carga $(0.25 \%$ de azul de bromofenol; $0.25 \%$ de xilenocianol; $30 \%$ de glicerol, en agua). 
Los perfiles de los amplicones se analizaron con el sistema universal de detección de mutaciones DCode (Bio-Rad Laboratories, Hercules, CA) usando geles de acrilamida de $16 \mathrm{~cm} \mathrm{x} 16 \mathrm{~cm}$, mediante un gel de acrilamida al $7 \%$ con un gradiente de desnaturalización de 30-60\%, con una relación de (37.5:1), que se filtraron a $0.45 \mu \mathrm{m}$. Para ello, se prepararon dos soluciones para la elaboración del gel de degradación de 30 al 60\%. La solución al 30\% se preparó utilizando $12.5 \mathrm{ml}$ de acrilamida/Bis 40\%, $2 \mathrm{ml}$ de TAE 50X, $12 \mathrm{ml}$ de formamida y $12.6 \mathrm{~g}$ de urea grado Biología Molecular (Sigma-Aldrich) y $60.9 \mathrm{ml}$ de agua mili Q por $100 \mathrm{ml}$. La solución al $60 \%$ contenía 12.5 $\mathrm{ml}$ de acrilamida/Bis 40\%, $2 \mathrm{ml}$ de TAE 50X, $24 \mathrm{ml}$ de formamida, $25.2 \mathrm{~g}$ de urea y $36.3 \mathrm{ml}$ de agua de mili Q por $100 \mathrm{ml}$.

Ambas soluciones fueron filtradas a través de un filtro de $0.45 \mu \mathrm{m}$ y desgasificadas. La formación del gel se llevó a cabo por el formador de gradientes BioRad 475, que fue cargado con $20 \mathrm{ml}$ de solución desnaturalizante, $70 \mu \mathrm{l}$ de persulfato al 10\% y $10 \mu \mathrm{l}$ de TEMED La electroforesis del gel se realizó durante 16 horas en amortiguador TAE $(0.5 \mathrm{X}, \mathrm{pH} 8)$ a $60{ }^{\circ} \mathrm{C}$ utilizando una fuente de alimentación universal PowerPac (Bio-Rad), con un voltaje inicial de $200 \mathrm{~V}$ durante $10 \mathrm{~min}$, luego el voltaje se redujo en $85 \mathrm{~V}$ durante $16 \mathrm{~h}$ (Neilson et al., 2013). El gel fue teñido según la técnica descrita por Radojkovic y Kušic (2000). El procedimiento de tinción consistió en una fijación inicial de pre-tinción durante 3 min en etanol al 10\%, 0.5\% de ácido acético.

La tinción durante 5 min en solución de fijación más $0.2 \%$ de nitrato de plata, el lavado del gel en agua durante 2 min, y el desarrollo durante 5 min en $30 \%$ de $\mathrm{NaOH}$ y $0.1 \%$ de formaldehído en agua de Milli-Q. Una vez corrido el gel, se recortaron las bandas identificadas y se realizó una extracción de 5 min. Con agua y agitación. Se aplicó un gel de agarosa al 30\% (80 V, 45 min) para corroborar la presencia de ADN y luego se realizó la secuenciación por el Macrogeno.

La historia evolutiva se dedujo utilizando el método de Máxima Verosimilitud basado en el modelo Kimura de dos parámetros (Kimura, 1980), seleccionado previamente ocho mediante un criterio de información bayesiano. Se muestra el árbol con la mayor probabilidad de tronco (-1499). Junto a las ramas se muestra el porcentaje de árboles en los que se agruparon los taxones asociados.

Los árboles iniciales para la búsqueda heurística se obtuvieron automáticamente aplicando los algoritmos Neighbor-Join y BioNJ a una matriz de distancias por pares estimadas utilizando el enfoque de máxima erosimilitud compuesta (MCL) y luego seleccionando la topología con valor de logaritmo de verosimilitud superior. El árbol se dibuja a escala, con las longitudes de las ramas medidas en el número de sustituciones por sitio. En el análisis se utilizaron 32 secuencias de nucleótidos. Se eliminaron todas las posiciones que contenían lagunas y datos faltantes. Había 239 posiciones en el conjunto de datos finales. Los análisis evolutivos se realizaron en MEGA7 (Kumar et al., 2016).

\section{Medición de la actividad enzimática}

Las actividades de las enzimas extracelulares, pectinasa, celulasa y xilanasa, se cuantificaron mediante la liberación de azúcares reductores. Las reacciones se llevaron a cabo en un amortiguador de citratos (50 mmol, $\mathrm{pH}$ 5.5). La actividad pectinasa se midió utilizando $1 \%$ de pectina de abedul incubada a $37{ }^{\circ} \mathrm{C}$ durante 30 minutos (Zhang et al., 2000). La actividad celulasas se cuantificó utilizando $2 \%$ de carboximetilcelulosa incubada a $50^{\circ} \mathrm{C}$ durante 30 minutos (Ghose, 1987). 
La actividad xilanasa se midió utilizando un $1 \%$ de xilano de madera de abedul incubado a $50{ }^{\circ} \mathrm{C}$ durante 15 minutos (Loera y Córdova, 2003). Todos los volúmenes de reacción fueron: $750 \mathrm{ml}$ de sustrato con $250 \mathrm{ml}$ de extracto enzimático, mezclado en una agitación orbital de $250 \mathrm{rpm}$. Las soluciones se midieron a una longitud de onda de $540 \mathrm{~nm}$ con un espectrofotómetro Beckman ${ }^{\circledR}$ DU 640.

La actividad de la proteasa se determinó utilizando un método modificado de Alef y Nannipieri (1995). Se añadió una alícuota de $1 \mathrm{ml}$ de extracto de enzima a $5 \mathrm{ml}$ de solución de caseína al 2\% y se incubó a $50{ }^{\circ} \mathrm{C}$ bajo agitación durante $2 \mathrm{~h}$. Las actividades enzimáticas se expresaron como U por gramo de materia seca, donde 1 unidad (U) es la cantidad de enzima que cataliza la generación de $1 \mu \mathrm{mol}$ de producto por minuto bajo las condiciones de reacción enzimática.

\section{Análisis estadístico}

Todas las mediciones y pruebas se realizaron por triplicado. Se realizó un análisis de variables múltiples $(\alpha=0.05)$ y el análisis de los componentes principales utilizando Statgraphics ${ }^{\circledR}$ Centurion XVII.

\section{Resultados y discusión}

\section{Efecto de la aireación durante el compostaje}

Para analizar el efecto de la tasa de aireación (vkgm), se evaluó el contenido de $\mathrm{CO}_{2}$ (v/v) producido en todas las condiciones Figura 1. La generación de $\mathrm{CO}_{2}$ fueron 31, 20, 14, 9, y $2 \%$ a 0.032, 0.064, $0.125,0.251$, y 0.392 vkgm, respectivamente. Un análisis de componentes principales (ACP) se muestra en la Figura 2a. El ACP mostró que un grupo principal que contenía el CR acumulado, el CR instantáneo, la pectinasa y el $\mathrm{CO}_{2}$ como variables agrupadas, que están asociadas entre si y otro grupo principal contenía, azúcares reductores, xilanasa y celulasa (Figura 2a). Además, se observó que la vkgm y la proteasa están correlacionadas negativamente con la pectinasa, el $\mathrm{CO}_{2}$ y el CR.
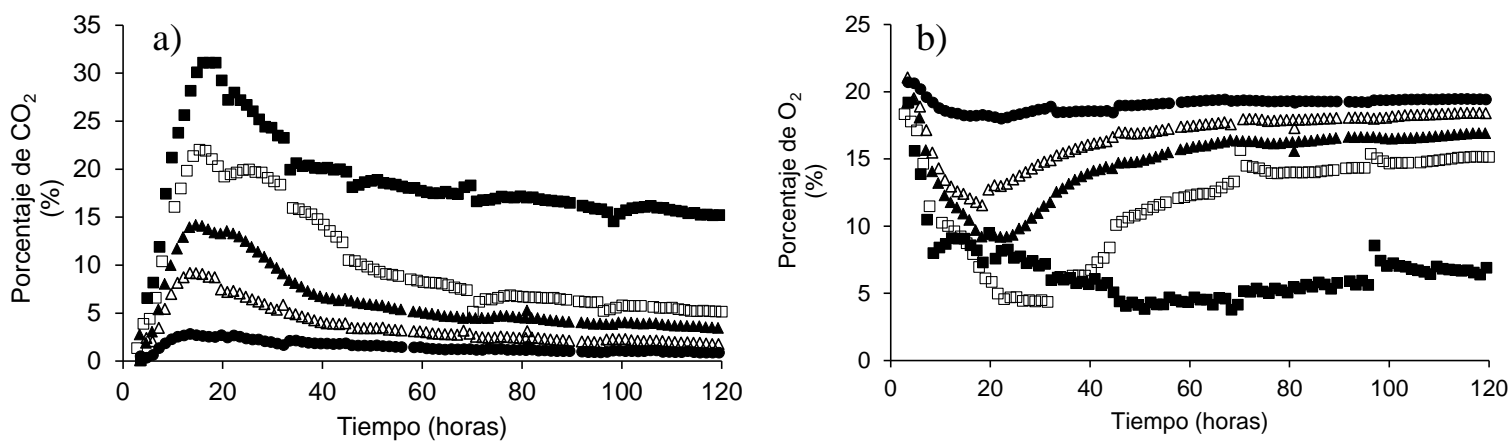

Figura 1. Concentración de a) $\mathrm{CO}_{2} ;$ y b) $\mathrm{O}_{2}$ en la salida gaseosa durante la degradación aerobia del FORSU a diferentes velocidades de aireación $($ vkgm) $\square$ 0.032, $\square 0.064, \Delta \mathbf{0 . 1 2 5}, \Delta 0.201$ y $\bullet$ 0.392). 

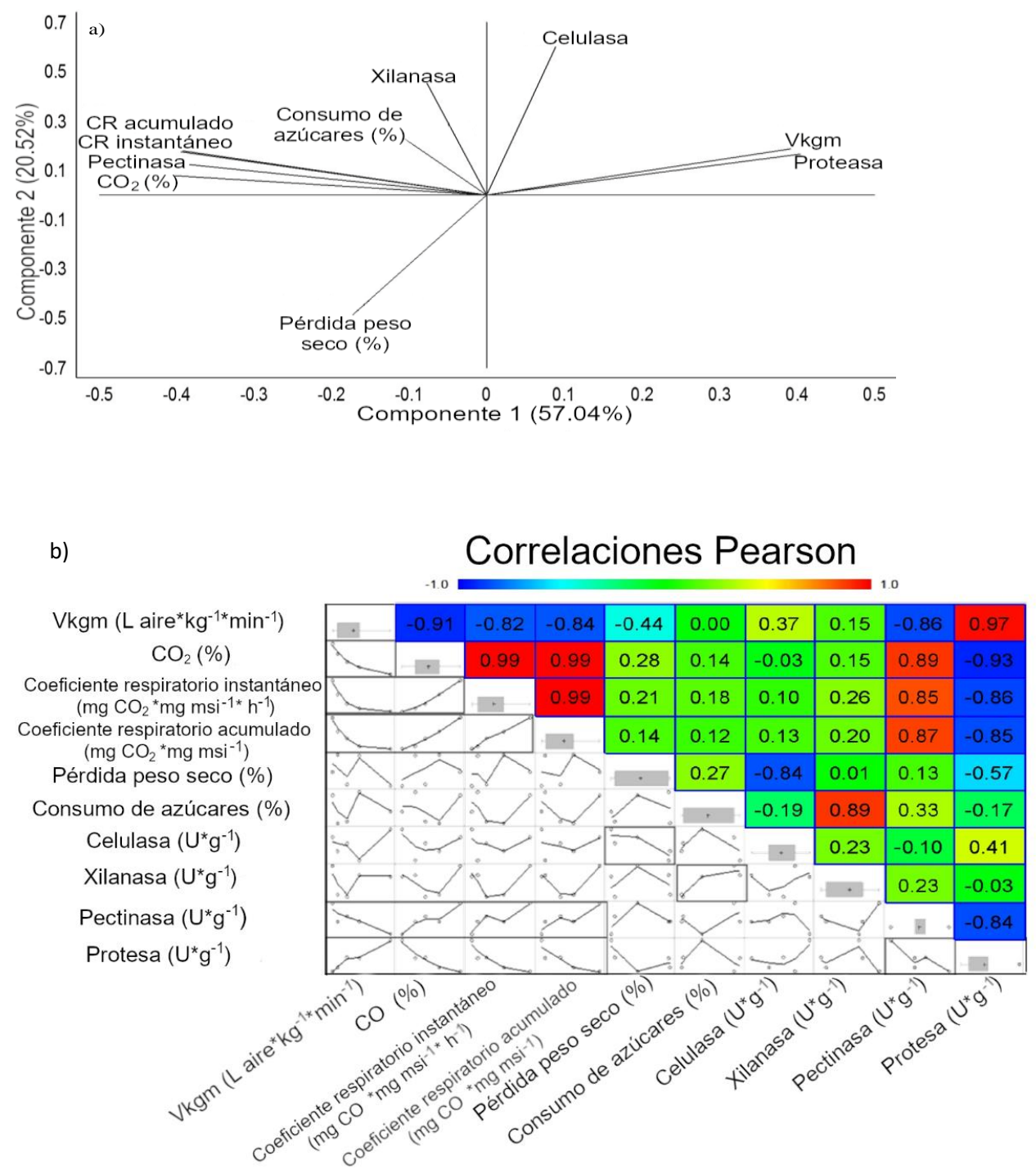

Figura 2. a) análisis de componentes principales (ACP); y b) matriz de correlación y coeficientes de correlación de Pearson entre la tasa de aireación, respuestas fisicoquímicas, respirométricas y enzimáticas determinadas

El análisis demostró que un modelo de 3 componentes explica $96.34 \%$ de la variabilidad y un modelo de 2 componentes explica 76\%. Una matriz de dispersión y los coeficientes de correlación de Pearson entre cada par de variables se incluyen en la Figura 2b. Se observó que la tasa de aireación (vkgm) tiene una correlación negativa significativa con respecto a la actividad de las pectinasas (-0.86) y con las respuestas de la actividad respirométrica como el contenido de $\mathrm{CO}_{2}$ (0.91), el CR instantáneo (-0.82) y el CR acumulado (-0.84). Por otra parte, la vkgm tiene una correlación positiva significativa con la actividad de las proteasas (0.97).

Además, la alta actividad de las proteasas indica que las proteínas solubles son degradadas por las proteasas y podría ser consecuencia de que la actividad pectinasa se reduce como resultado. La actividad proteasa tiene una correlación positiva significativa con la actividad celulasa (0.41). Estas 
observaciones también fueron reportadas por Goyal et al. (2005); He et al., (2013), que observaron un aumento de la actividad de la proteasa y la celulasa durante la fase activa del compostaje. Kayikçioğlu et al. (2011) reportaron que la actividad proteasa máxima coincidió con la actividad microbiana máxima.

La ACP reveló que la pectinasa tiene una correlación positiva significativa con los índices respirométricos, a saber, el $\mathrm{CO}_{2}(0.89)$, el CR instantáneo (0.85) y el CR acumulado (0.87) y una correlación negativa con el vkgm (-0.86). Mientras tanto, las proteasas tienen una correlación negativa significativa a los índices respirométricos como el $\mathrm{CO}_{2}(-0.93)$, la CR instantánea (-0.86) y la CR acumulativa (-0.85) y una correlación positiva significativa a la vkgm (0.97).

Lo anterior confirma que la proteasa está correlacionada con las condiciones aerobias, mientras que la pectinasa sólo está presente en condiciones anaerobias. Puyuelo et al. (2011); Xu et al. (2012) han señalado que el aumento de la actividad enzimática depende de los consorcios microbianos presentes y de la concentración de microorganismos. Por consiguiente, el cambio en la actividad de la proteasa y la pectinasa podría indicar cambios en los consorcios microbianos durante el proceso.

\section{Microorganismos presentes en diferentes condiciones de aireación}

La identificación de bacterias (Figura 3) Citrobacter freundi, Klebsiella oxytoca puede ser encontrada. Klebsiella oxytoca ha sido reportada como un alto productor de enzimas de celulosa (Golias et al., 2002). En las bajas tasas de aireación (0.032, 0.064 y $0.125 \mathrm{vkgm}) \mathrm{se}$ ha puesto de manifiesto la presencia de Enterobacterias, lo que se relaciona con actividades de baja proteasa y alta pectinasa (es decir, la prevalencia de entornos semianerobios). Estas bacterias son quimioorganotróficas con un metabolismo tanto respiratorio como fermentativo. La mayoría son oxidasas negativas, excepto la Plesiomonas. La mayoría reducen el nitrato a nitrito, excepto las fermentaciones de Saccharobacter y algunas cepas de Erwinia y Yersinia (Brenner y Farmer, 2015).

Algunas cepas, como E. cloacae, E. hafniae o E. aerogenes son productoras de lipasas. Por otro lado, Buttiauxella sp. Es conocido como productor de fitasa y un anaerobio facultativo de la familia de las Enterobacteriacea (Mitra et al., 2010). En la condición con la mayor tasa de aireación probada (0.392 y $0.251 \mathrm{vkgm})$, las bacterias Acinetobacter fueron las más representativas, siendo estrictamente aerobias con actividad catalasa positiva y oxidasa negativa. La mayoría de las cepas pueden crecer en un medio mínimo simple con una sola fuente de carbono y una temperatura de incubación entre $20-30{ }^{\circ} \mathrm{C}$.

Lewin et al. (2016); Wang et al. (2016) mencionaron que Actinobacter contribuye al ciclo mundial del carbono mediante la descomposición de la biomasa de las plantas. Sus enzimas pueden contribuir a la descomposición a escala industrial de la biomasa vegetal celulósica en azúcares simples que podrían convertirse en biocombustibles. Actinobacter alberga un catálogo sustancialmente amplio de los genes de cellobiohidrolasa, $\beta$-glucosidasa, acetil xilano esterasa, arabinofuranosidasa, pectina liasa y ligninasa. 


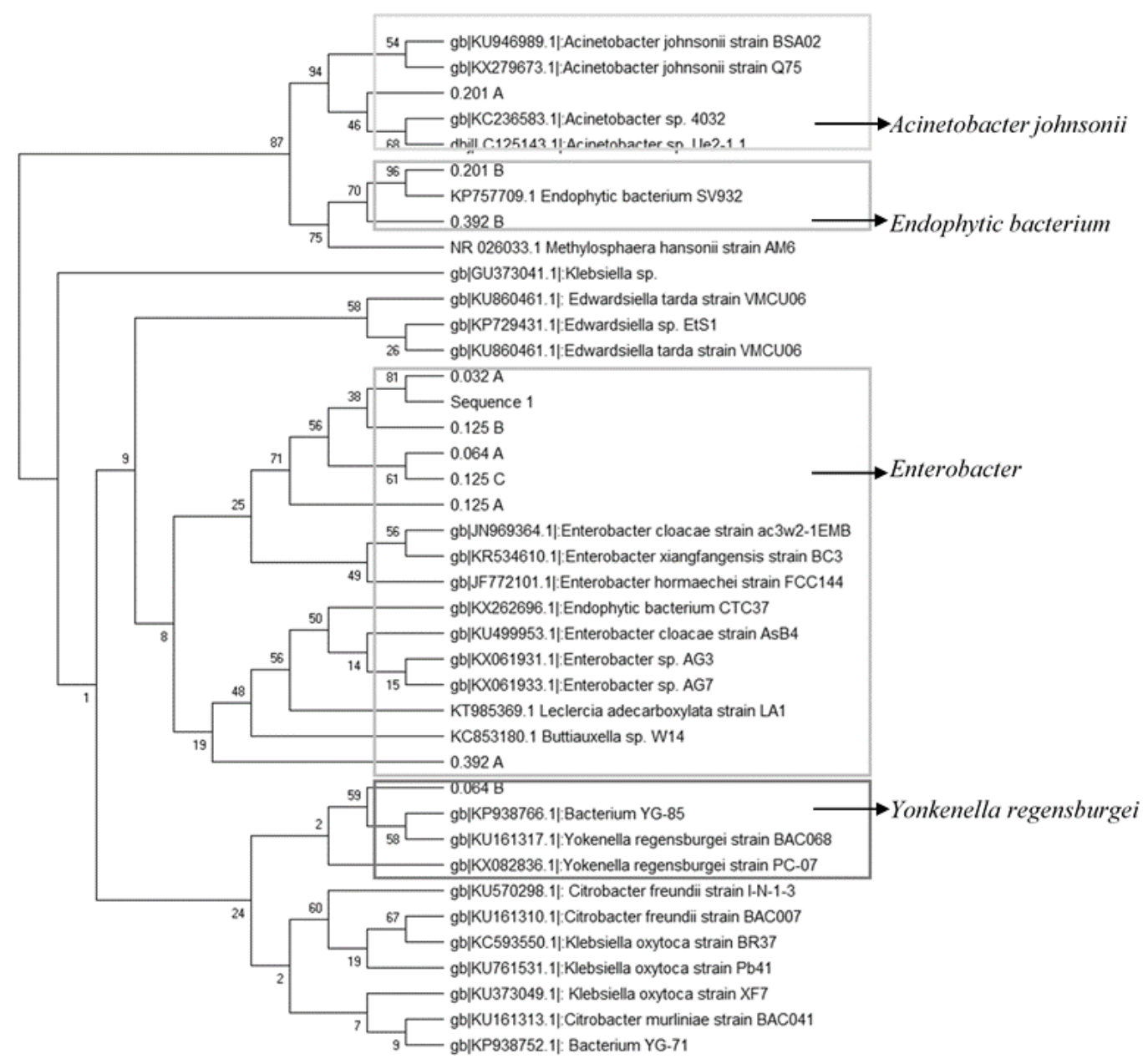

Figura 3. Análisis filogenético molecular por el método de máxima probabilidad. Las condiciones experimentales utilizadas fueron: inoculación por compostaje de PCBP, temperatura fijada a 35 ${ }^{\circ} \mathrm{C}$, humedad inicial 70\% (wb), pH 6, varias tasas de aireación, a saber, 0.032, 0.064, 0.125, 0.251 y 0.392 vkgm. Las muestras se obtuvieron en el momento de máxima tasa de generación de $\mathrm{CO}_{2}$.

\section{Conclusiones}

Los resultados revelaron una fuerte correlación positiva entre la actividad xilanasa, pectinasa y la pérdida de peso seco, mientras que todas las actividades enzimáticas aumentaron a mayores tasas de aireación. En este sentido, el alto consumo de azúcares reductores se correlacionó únicamente con la actividad de la xilanasa y la pectinasa, ya que la celulasa y la proteasa tenían una actividad menor. La EGGD reveló que en las tasas de aireación más altas $(0.392$ y 0.251 vkgm), la presencia de Actinobacter era dominante. Por lo cual, se puede inferir que bajo un sistema en condiciones aerobias la presencia de Actinobacter favorece a la degradación de la materia orgánica.

\section{Literatura citada}

Alef, K. and Nannipieri, P. 1995. Chapter 7-Enzyme activities. In: Methods in Applied Soil Microbiology and Biochemistry. Kassem, A.; Paolo, N. (eds). $1^{\text {st }}$ Edition. Academic Press. London. 311- 373 pp. 
ASTM (American Society for Testing and Materials) D5231-92. 2016. Standard Test Method for Determination of the Composition of Unprocessed Municipal Solid Waste. ASTM International. West Conshohocken. Pensilvania. USA. 6 p. https://doi.org/10.1520/ D523192R16.

Bayard, R.; Gonzalez-Ramirez, L.; Guendouz, J.; Benbelkacem, H.; Buffière, P. and Gourdon, R. 2015. Statistical analysis to correlate bio-physical and chemical characteristics of organic wastes and digestates to their anaerobic biodegradability. Waste and biomass valorization. 6 (5), 759-769. https://doi.org/10.1007/s12649-015-9411-2.

Bernal, M. P.; Alburquerque, J. A. and Moral, R. 2009. Composting of animal manures and chemical criteria for compost maturity assessment. A review Bioresource Technology. 100(22):5444-5453. https://doi.org/10.1016/j.biortech.2008.11.027.

Brenner, D. J. and Farmer III, J. J. 2005. Family I. Enterobacteriaceae. In: Bergey's Manual of Systematic Bacteriology. Brenner D. J.; Krieg N. R.; Staley J. T. ; Garrity G. M.; Boone, D. R.; Vos, P.; Goodfellow, M.; Rainey, F. A. and Schleifer, K. H. (eds). $2^{\text {nd }}$ ed. Springer. New York, USA. 587-607 pp. https://doi.org/10.1002/9781118960608.fbm00222.

El Achkar, J. H.; Lendormi, T.; Hobaika, Z.; Salameh, D.; Louka, N.; Maroun, R. G. and Lanoisellé, J. L. 2017. Anaerobic digestion of nine varieties of grape pomace: correlation between biochemical composition and methane production. Biomass Bioenergy. 107:335344. https://doi.org/10.1016/j.biombioe.2017.10.030.

Ghose, T. K. 1987. Measurement of cellulase activities. Pure Appl. Chem. 59(2):257-268. https://doi.org/10.1351/pac198759020257.

Gil, A.; Toledo, M.; Siles, J. A. and Martín, M. A. 2018. Multivariate analysis and biodegradability test to evaluate different organic wastes for biological treatments: anaerobic co-digestion and co-composting. Waste Management. 78:819-828. https://doi.org/10.1016/j.wasman. 2018.06.052.

Golias, H.; Dumsday, G. J.; Stanley, G. A. and Pamment, N. B. 2002. Evaluation of a recombinant Klebsiella oxytoca strain for ethanol production from cellulose by simultaneous saccharification and fermentation: comparison with native cellobiose-utilising yeast strains and performance in co-culture with thermotolerant yeast and zymomonas mobilis. J. Biotechnol. 96(2):155-168. https://doi.org/10.1016/S0168-1656 (02)00026-3.

Goyal, S.; Dhull, S. K. and Kapoor, K. K. 2005. Chemical and biological changes during composting of different organic wastes and assessment of compost maturity. Bior. Technol. 96(14):1584-1591. https://doi.org/10.1016/j.biortech.2004.12.012.

Guo, R.; Li, G.; Jiang, T.; Schuchardt, F.; Chen, T.; Zhao, Y. and Shen, Y. 2012. Effect of aeration rate, $\mathrm{C} / \mathrm{N}$ ratio and moisture content on the stability and maturity of compost. Bior. Technol. 112:171-178. https://doi.org/10.1016/j.biortech.2012.02.099.

He, Y.; Xie, K.; Xu, P.; Huang, X.; Gu, W.; Zhang, F. and Tang, S. 2013. Evolution of microbial community diversity and enzymatic activity during composting. Res. Microbiol. 164(2):189-198. https://doi.org/10.1016/j.resmic.2012.11.001.

Kayikçioğlu, H. H. and Okur, N. 2011. Evolution of enzyme activities during composting of tobacco waste. Waste Manag. Res. 29(11):1124-1133. https://doi.org/10.1177/0734242X 10392813.

Kimura, M. 1980. A simple method for estimating evolutionary rates of base substitutions through comparative studies of nucleotide sequences. J. Mol. Ev. 16(2):111-120. https://doi.org/10.1007/BF01731581. 
Komilis, D.; Kontou, I. and Ntougias, S. 2011. A modified static respiration assay and its relationship with an enzymatic test to assess compost stability and maturity. Bior. Technol. 102(10):5863-5872. https://doi.org/10.1016/j.biortech.2011.02.021.

Kumar, S.; Stecher, G. and Tamura, K. 2016. MEGA7: molecular evolutionary genetics analysis version 7.0 for bigger datasets. Mol. Biol. Evol. 33(7):1870-1874. https://doi.org/10.1093/ molbev/msw054.

Lewin, G. R.; Carlos, C.; Chevrette, M. G.; Horn, H. A.; McDonald, B. R.; Stankey, R. J. and Currie, C. R. 2016. Evolution and ecology of actinobacteria and their bioenergy applications. Annual Review MicrobioL. 70:235-254. https://doi.org/10.1146/annurevmicro-102215-095748.

Li, Z.; Lu, H.; Ren, L. and He, L. 2013. Experimental and modeling approaches for food waste composting: a review. Chemosphere. 93(7):1247-1257. https://doi.org/10.1016/j. chemosphere.2013.06.064.

Liang, C.; Das, K. C. and McClendon, R. W. 2003. The influence of temperature and moisture contents regimes on the aerobic microbial activity of a biosolids composting blend. Bior. Technol. 86(2):131-137. https://doi.org/10.1016/S0960-8524 (02)00153-0.

Liu, X.; Bayard, R.; Benbelkacem, H.; Buffière, P. and Gourdon, R. 2015. Evaluation of the correlations between biodegradability of lignocellulosic feedstocks in anaerobic digestion process and their biochemical characteristics. Biom. Bioen. 81:534-543. https://doi.org/10.1016/j.biombioe.2015.06.021.

Loera, O. and Córdoba, J. 2003. Improvement of xylanase production by a parasexual cross between Aspergillus niger strains. Braz. Archiv. Biol. Technol. 46(2):177-181. https://doi.org/10.1590/S1516-89132003000200006.

Martínez-Valdez, F. J.; Martínez-Ramírez, C.; Martínez-Montiel, L.; Favela-Torres, E.; Soto-Cruz, N. O.; Ramírez-Vives, F. and Saucedo-Castañeda, G. 2015. Rapid mineralisation of the organic fraction of municipal solid waste. Bior. Technol. 180:112-118. https://doi.org/10.1016/j.biortech.2014.12.083.

Martins, S.; Mussatto, S. I.; Martínez-Avila, G.; Montañez-Saenz, J.; Aguilar, C. N. and Teixeira, J. A. 2011. Bioactive phenolic compounds: production and extraction by solid-state fermentation. A review. Biotechnol. Adv. 29(3):365-373. https://doi.org/10.1016/j. biotechadv.2011.01.008.

Mitra, S.; Khare, S. K. and Singh, R. 2010. Alkaline lipase production from Enterobacter aerogenes by solid-state fermentation of agro-industrial wastes. International Journal of Environment and Waste Management, 5 (3-4), 410-418. https://doi.org/10.1504/IJEWM.2010.032017.

Neilson, J. W.; Jordan, F. L. and Maier, R. M. 2013. Analysis of artifacts suggests EGGD should not be used for quantitative diversity analysis. J. Microbiol. Methods. 92(3):256263.https://doi.org/10.1016/j.mimet.2012.12.021.

Puyuelo, B.; Ponsá, S.; Gea, T. and Sánchez, A. 2011. Determining C/N ratios for typical organic wastes using biodegradable fractions. Chemosphere. 85(4):653-659. https://doi.org/10. 1016/j.chemosphere.2011.07.014.

Radojkovic, D. and Kušic, J. 2000. Silver staining of denaturing gradient gel electrophoresis gels. Clinical Chem. 46(6):883-884.

Rasapoor, M.; Nasrabadi, T.; Kamali, M. and Hoveidi, H. 2009. The effects of aeration rate on generated compost quality, using aerated static pile method. Waste Management. 29(2):570-573. https://doi.org/10.1016/j.wasman.2008.04.012.

Raut, M. P.; Prince-William, S. P; Bhattacharyya, J. K.; Chakrabarti, T. and Devotta, S. 2008. Microbial dynamics and enzyme activities during rapid composting of municipal solid waste-a compost maturity analysis perspective. Bior. Technol. 99(14):6512-6519. https://doi.org/10.1016/j.biortech.2007.11.030. 
Rodríguez-Fernández, D. E.; Rodríguez-León, J. A.; de-Carvalho, J. C.; Karp, S. G.; Sturm, W.; Parada, J. L. and Soccol, C. R. 2012. Influence of airflow intensity on phytase production by solid-state fermentation. Bior. Technol. 118:603-606. https://doi.org/10.1016/j.biortech. 2012.05.032.

Saludes, R. B.; Iwabuchi, K.; Kayanuma, A. and Shiga, T. 2007. Composting of dairy cattle manure using a thermophilic-mesophilic sequence. Biosystems Engineering. 98(2):198-205. https://doi.org/10.1016/j.biosystemseng.2007.07.003.

Saucedo-Castañeda, G.; Favela-Torres, E.; Viniegra-González, G.; Torres-Mancera, M. T.; Figueroa-Montero, A. and Rosales-Zamora, G. 2013. Respirometry system with remote management for the on-line monitoring of the concentration of $\mathrm{CO}_{2}$ and $\mathrm{O}_{2}$ and flow of the exhausting gases in biological processes. Mexican patent 336733 granted January 22 th, 2016.

Tiquia, S. M. 2002. Evolution of extracellular enzyme activities during manure composting. J. Appl. Microbiol. 92(4):764-775. https://doi.org/10.1046/j.1365-2672.2002.01582.x.

Toledo, M.; Siles, J. A.; Gutiérrez, M. C. and Martín, M. A. 2018. Monitoring of the composting process of different agroindustrial waste: Influence of the operational variables on the odorous impact. Waste Management. 76:266-274. https://doi.org/10.1016/j.wasman. 2018.03.042.

Torres-Mancera, M. T.; Figueroa-Montero, A.; Favela-Torres, E.; Rosales-Zamora, G.; Nampoothiri, K. M. and Saucedo-Castañeda, G. 2018. Online monitoring of solid-state fermentation using respirometry. In current developments in biotechnology and bioengineering. Elsevier. 97-108 pp. https://doi.org/10.1016/B978-0-444-639905.00006-2.

Wang, C.; Dong, D.; Wang, H.; Müller, K.; Qin, Y.; Wang, H. and Wu, W. 2016. Metagenomic analysis of microbial consortia enriched from compost: new insights into the role of Actinobacteria in lignocellulose decomposition. Biotechnol. Biofuels. 9(1):22-31. https://doi.org/10.1186/s13068-016-0440-2.

Xu, S. Y.; Karthikeyan, O. P.; Selvam, A. and Wong, J. W. C. 2012. Effect of inoculum to substrate ratio on the hydrolysis and acidification of food waste in leach bed reactor. Bior. Technol. 126:425-430. https://doi.org/10.1016/j.biortech.2011.12.059.

Zhang, J.; Henriksson, G. and Johansson, G. 2000. Polygalacturonase is the key component in enzymatic retting of flax. J. Biotechnol. 81(1):85-89. https://doi.org/10.1016/S0168-1656 (00)00286-8. 